\title{
Detection of Optic Disc in Retina using Hough Transform
}

Vijaya Patil, Vaishali Kumbhakarna, Dr. Seema Kawathekar Department of Computer Science, Dr. Babasaheb Ambedkar Marathwada University, Aurangabad vijaya.cse.msc@gmail.com,vmk_17@yahoo.co.in,seema_babrekar@yahoo.co.in

\section{ABSTRACT}

We propose a method to automatically locate the Optic Disc (OD) in fundus images of the retina. Based on the properties of the OD, our proposed method includes edge detection using the Canny method, and detection of circles using the Hough transform. The Hough transform assists in the detection of the center and radius of a circle that approximates the margin of the OD. Based on the feature that the OD is one of the brightest areas in fundus image, the potential circles can be detected by Hough transform.

\section{Indexing terms/Keywords}

Hough transform, Optic Disc.

\section{Academic Discipline And Sub-Disciplines}

Computer Science; Image Processing; Retinopathy; Medical Applications.

\section{SUBJECT CLASSIFICATION}

Computer Science; Image Processing.

\section{TYPE (METHOD/APPROACH)}

Proposal of a new method.

\section{Council for Innovative Research}

Peer Review Research Publishing System

Journal: INTERNATIONAL JOURNAL OF COMPUTERS \& TECHNOLOGY

Vol. 15 , No. 3

www.ijctonline.com, editorijctonline@gmail.com 


\section{INTRODUCTION}

Retina is the innermost layer of the eye which can be visualized using an apparatus such as fundus camera. The two main structures used in retinal image analysis are blood vessels and optic disc. Optic disc is the brightest region in the retinal image and the blood vessels originate from its center [1].

The information about the optic disk can be used to examine severity of some diseases like glaucoma. Changes in the optic disk can indicate the current state and progression of a certain disease. The location of the optic disk is an important issue in retinal image analysis as it is a significant landmark feature. The diameter of an optic disk is usually used as a reference length for measuring distances and sizes of circles [2].

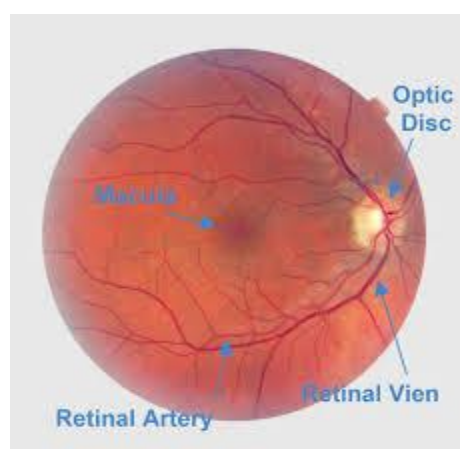

Figure 1. Retina

An important pre-requisite for automation is the accurate localization of the main anatomical features in the image. An accurate and efficient detection of these structures is a significant task in an automated retinal image analysis system. Figure 1 shows the retinal fundus image with main anatomical structures. The retina is an interior surface of human eye. It converts light rays into electrical signals and sends them to the brain through optic nerves. Optic nerve is the cable connecting eye to the brain. OD is the brightest region within the retinal image [3].

The OD has an inner portion called the optic cup. The optic cup is always smaller than the disc. The relative size of optic cup to disc is called cup disc ratio (CDR). The CDR ranges from 0.1 to 0.5 [4] [5]. Specifically, this is an important indicator for glaucoma [6].

\section{DATABASE OVERVIEW}

In this section, we briefly explain about the database used for our analysis. The database is freely downloadable and used for scientific research purposes. DIARET DBO is a public database for benchmarking diabetic retinopathy. The current database consists of 130 color fundus images of which 20 are normal and 110 contain signs of the diabetic retinopathy. Images were captured with a 50 degree field-of-view digital fundus camera with random camera settings. The data corresponds to practical situations, and can be used to evaluate the general performance of diagnosis methods. This data set is referred to as "calibration level 0 fundus images".

DIARET DB1 is a public database for benchmarking diabetic retinopathy. The database consists of 89 colour fundus images of which 84 contain at least mild non-proliferative signs (Micro aneurysms) of the diabetic retinopathy, and 5 are considered as normal which do not contain any signs of the diabetic retinopathy according to all experts who participated in the evaluation. Images were captured using the same 50 degree field-of-view digital fundus camera with varying imaging settings. The data corresponds to a good (not necessarily typical) practical situations, where the images are comparable, and can be used to evaluate the general performance of diagnostic methods. This data set is referred to as "calibration level 1 fundus images".

\section{PROPOSED APPROACH}

In this section, we outline our approach to automatically locate the OD in fundus images of the retina. We divide our approach into two stages: A) Pre-processing stage and B) Optic disc detection. Figure 2 shows the output of preprocessing stage.

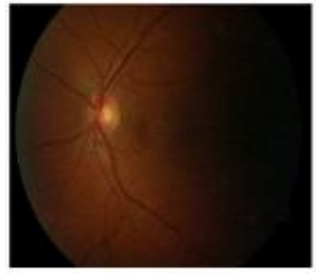

a) Original image

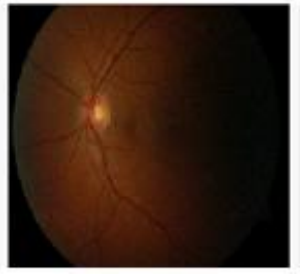

b) Resized image

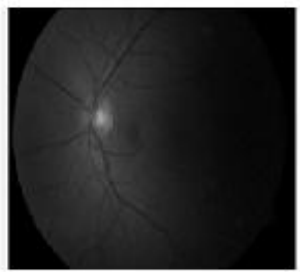

c) Grey image

Figure 2. Pre-processing stage 


\section{A) Pre-processing stage}

This stage is sub-divided into image acquisition and image filtering.

a. Image acquisition

All digital retinal images are taken from retinal fundus camera. The images are stored in JPEG image format file (.jpg) the original (RGB) image is transformed into appropriate colour space for further processing. The image is resized and later filtering technique is used to reduce the effect of noise. After using the filtering technique, the noise such as salt and pepper are removed from the image.

b. Median filter

The median filter is a non-linear filter type which is used to reduce the effect of noise without blurring sharp edges. The working principle of the median filter is to first arrange the pixel values in either the ascending or descending order and then compute the median value of neighborhood pixels.

\section{B) Optic disc detection}

The center and boundary of the optic disc are found by applying the Hough transform to the gradient image. The Hough transform is a method for finding shapes in an image. The basic idea behind the Hough transform is to transform the image into a parameter space that is constructed specifically to describe the desired shape analytically. Maxima in this parameter space then correspond to the presence of the desired shape in image space.

The circular Hough transform is almost identical to the Hough transform for lines, but uses the parametric form for a circle as denoted in equation (1),

$$
(x-a)^{2}+(y-b)^{2}+r^{2}=0
$$

where $(a, b)$ is the centre of the circle of radius $r$ that passes through $(x, y)$. The Hough space is three dimensional (3D). The gradient image is transformed to a set of three parameters, representing the accumulator, its center and its radius. For each feature point, votes are accumulated in an accumulator array for all parameter combinations.

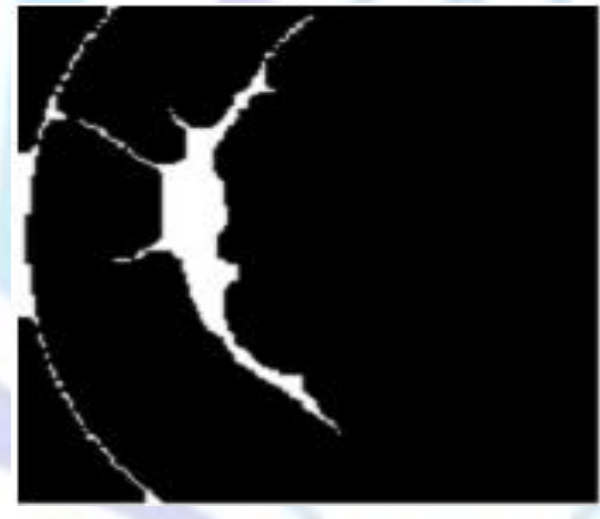

a) Canny detection

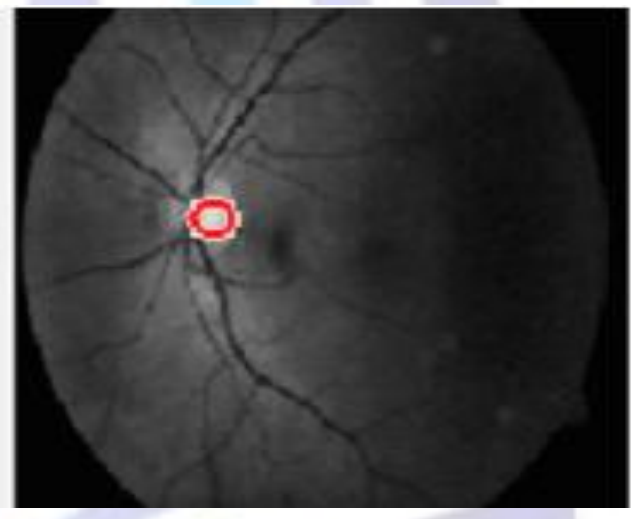

b) Detection of OD

Figure 3: Optic disc detection.

The accumulator will have set of edge points; each edge points contribute a circle of radius $r$ in the accumulation space. The accumulation space has a peak where these contributory circles overlap at the centre of the original circle. The centre is an $\mathrm{N} \times 2$ matrix with each row containing the $(\mathrm{x}, \mathrm{y})$ positions of the circles detected in the image. The estimated radius of the circles detected is stored in an $\mathrm{N} \times 1$ column vector with a one-to-one correspondence to the center array. The corresponding circle can then be plotted over the original fundus image [1]. Figure 3 shows the Canny detection and OD detection. Figure 4 shows general flow diagram for OD detection. 


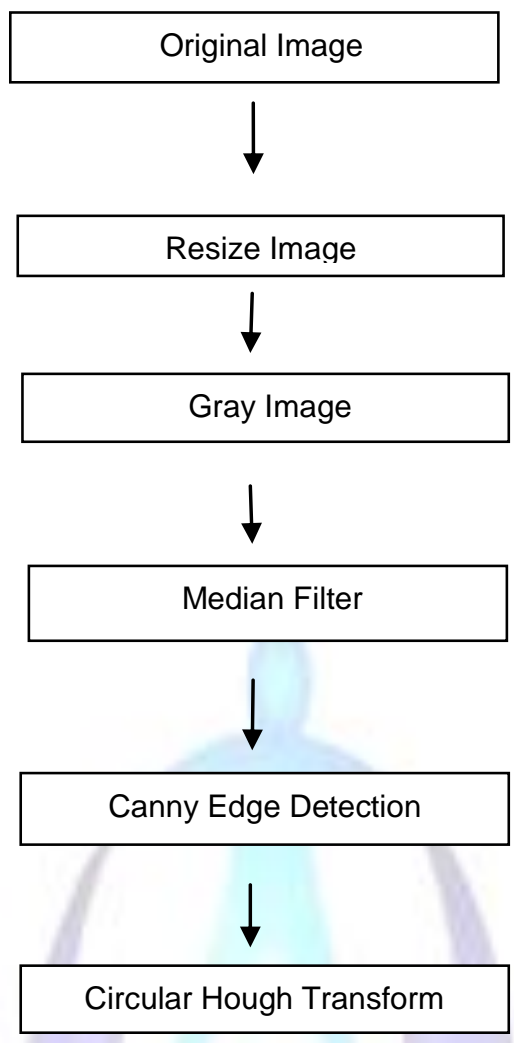

Figure 4. Flow diagram of detection of optic disc.

\section{EXPERIMENTS AND RESULTS}

In this section, we explain the experiments and results obtained.

\begin{tabular}{|c|c|c|c|}
\hline Test database & Images & OD Detected & \% Accuracy \\
\hline Diaretdb0 & 130 & 126 & 96.92 \\
\hline Diaretdb1 & 89 & 86 & 96.62 \\
\hline
\end{tabular}

Table I: Results of the experiments.

We conduct experiments on the freely available database as mentioned in section II. We choose 130 images from Diaretdb0 and 89 images from Diaretdb1. We employ our approach as per section III and detect ODs from the images. Table I shows the number of images taken for our experiments and detected ODs. Using our approach, we hit around

\begin{tabular}{|c|c|c|c|}
\hline Directbd0 & x co-ordinate & y co-ordinate & Radius \\
\hline image001 & 179.2323556 & 80.97328582 & 14.039 \\
\hline imag002 & 91.00961589 & 121.6833585 & 13.27811897 \\
\hline image003 & 180.0800319 & 128.9727305 & 13.89564821 \\
\hline image004 & 85.67543618 & 137.8075234 & 14.11034677 \\
\hline image008 & 65.10749429 & 120.3164019 & 12.31958147 \\
\hline image010 & 58.34443815 & 112.9221488 & 15.340573179 \\
\hline
\end{tabular}

Table II. Images with radius of the OD and $(x, y)$ co-ordinates.

$96 \%$ of accuracy. Table II shows few images in which OD has been detected, and shows the $x$ and $y$ co-ordinates of the OD along with the radius. 


\section{CONCLUSION}

Circular Hough transform method is used for optic disc detections, the input images are taken from DIARETDB0 and DIARETDB1. The input image is in RGB color space and for the further processes the image is converted into appropriate color space. The median filter is used for the noise reduction without blurring the edge. And then Canny edge detection method is used. The results of our work can play a major role in the screening of eye diseases.

\section{REFERENCES}

[1] A. Dehghani, H. Moghaddam, and S. Moin, "Optic disc localization in retinal images using histogram matching," EURASIP Journal on Image and Video Processing, vol. 2012, no. 19, pp. 1687--5281, 2012.

[2] S. Sekhar, W. Nuaimy, and A. Nandi, "Automated localization of retinal optic disc using Hough transform," IEEE International Symposium on Biomedical Imaging: From Nano to Macro, 2008.

[3] D. Godse and D. Bormane, "Automated localization of optic disc in retinal images," International Journal of Advanced Computer Science and Applications, vol. 4, no. 2, 2013.

[4] P. Healey, P. Mitchell, W. Smith, and J. Wang, "Relationship between cup-disc ratio and optic disc diameter," Australian and New Zeland Journal of Ophthalmology, 1997.

[5] G. Joshi, J. Sivaswamy, K. Karan, and S. Krishnadas, "Optic disc and cup boundary detection using regional information," IEEE International Symposium on Biomedical Imaging: From Nano to Macro, 2010.

[6] S. Lavanya, "Detection of anatomical structures in optical fundus images," International Journal of Application or Innovation in Engineering \& Managemen, vol. 2, no. 5, pp. 430-435, 2013. 